\title{
The common quantitative genetic basis of wing morphology and diapause occurrence in the cricket Gryllus veletis
}

\author{
M Bégin ${ }^{1}$ and DA Roff ${ }^{2}$ \\ ${ }^{1}$ Department of Biology, McGill University, Montréal, Québec, Canada; ${ }^{2}$ Department of Biology, University of California-Riverside, \\ CA, USA
}

\begin{abstract}
A covariation between wing morphology and diapause occurrence has been observed in many insect species, but the genetic basis of this covariation has never been established. This study measures the heritability of, and genetic correlation between, these two ecologically important threshold traits in the cricket Gryllus veletis. A total of 81 fullsib families were reared in the laboratory to estimate these parameters. A comparison of laboratory and field samples showed that these two traits are highly plastic. The heritability of wing morphology was $0.25(0.09)$, the heritability of diapause occurrence was $0.77(0.11)$ and the genetic correlation between them was $0.61(0.19)$. These estimates did
\end{abstract}

not differ between males and females. The significance of these quantitative genetic parameters is discussed with reference to the monomorphism of natural populations of $G$. veletis for diapause occurrence and with reference to the trade-off between the ability to disperse by flight and the ability to diapause found in at least one closely related species. A survey of the literature reveals that genetic correlations between diapause occurrence or wing morphology and various other traits are common in insects, suggesting that these two traits are often genetically integrated in insect life-histories.

Heredity (2002) 89, 473-479. doi:10.1038/sj.hdy.6800168

Keywords: environmental heterogeneity; genetic correlation; heritability; threshold trait; trade-off; wing dimorphism

\section{Introduction}

Environmental heterogeneity in time and in space are important causes of life-history evolution in the wild (Tauber et al, 1986; Roff, 2002). Adaptations to a heterogeneous environment usually allow an organism to take advantage of favourable environmental units (temporal or spatial) while escaping harsh ones. These adaptations can be found in all life forms and at all scales, from the desiccation resistant seeds in desert plants to the seasonal migrations in birds. Two such traits, wing dimorphism and diapause occurrence, have been abundantly studied in insects (Dingle, 1978; Harrison, 1980; Roff, 1986a; Tauber et al, 1986; Danks, 1987; Zera and Denno, 1997). These two traits vary dichotomously at the phenotypic level, making them easy to study, but have a polygenic basis, making them relevant to the study of quantitative inheritance in nature. The evolution of this type of trait (reviewed in Roff, 1996) can be understood using the threshold model of quantitative genetics (Falconer and Mackay, 1996; Roff, 1997).

Diapause is a physiological process which allows an organism to survive a hostile period of the year, such as winter in northern latitudes or the dry season in arid areas, by becoming dormant. This process synchronises

Correspondence: M Bégin, Department of Biology, University of California-Riverside, Riverside, CA, USA, 92 521.E-mail: mbegin1@pobox.mcgill.ca

Received 27 December 2001; accepted 22 July 2002 growth and reproduction with predictably favourable seasons and is one of the most common adaptations to temporal heterogeneity among insects. Diapause is usually expressed as a state of low metabolic activity that is associated with a set of physiological and behavioural changes facilitating resistance to harsh environmental conditions (Tauber et al, 1986; Danks, 1987). This form of dormancy is partly regulated by environmental cues associated with the predictable arrival of some specific environmental conditions and generally occurs at a specific stage of the development of the organism (Tauber et al, 1986; Danks, 1987). In species in which diapause occurrence is variable, populations consist of direct developers and of diapausing individuals. Such variation can sometimes produce more than one pattern of voltinism within a population, as in the case of the cricket Allonemobius fasciatus (Mousseau and Roff, 1989). Diapause occurrence has been found to be heritable in several Arthropods (reviewed in Roff, 1996).

Flight is very common in insects and is one of the key adaptations of this class. The ability to fly is a very effective means of coping with heterogeneity in space, allowing organisms to actively find favourable environmental units either on a small or large geographical scale. Despite the usefulness of flight, wing dimorphism is common among insects (Harrison, 1980; Roff, 1986a; Zera and Denno, 1997). Populations of wing dimorphic insects typically consist of two distinct types of individuals: one with long wings and a generally functional flight apparatus (macropterous) and the other with short wings and 
an inability to fly (micropterous). Wing dimorphism is typically evolutionarily maintained by a compensation in other fitness components, a situation exemplified by the trade-off between flight and fecundity found in many insect species (Roff and Fairbairn, 1991). Wing morphology has been shown to be heritable in various Orthopteran species (reviewed in Roff and Fairbairn, 2001).

Genetic variation in wing morphology and in diapause occurrence are usually studied independently of each other (but see Dingle, 1978 for exceptions), probably because these two traits are two evolutionarily alternative ways of adapting to environmental heterogeneity. However, wing morphology and diapause occurrence have been found to be phenotypically correlated in the laboratory and/or in nature in many species of Heteropterans (Harrison, 1980; Harada and Numata, 1993) and Orthopterans (Sellier, 1954; Alexander, 1968; Tanaka, 1978). In addition Sellier (1954) performed physiological manipulations in the cricket Gryllus campestris and concluded that these two traits are mostly determined by a common developmental mechanism. This physiological evidence suggests that the determination of diapause occurrence and wing morphology might have a common genetic basis in insect species in which these two traits covary at the phenotypic level. If so, such pleiotropy could have been important during the evolution of the life cycles of these species.

The main objective of this study is to use quantitative genetics to investigate the common genetic basis of nymphal diapause occurrence and of wing morphology in the cricket $G$. veletis. Very little is known about the significance of the association between diapause occurrence and wing morphology and no attempt has yet been made to estimate the genetic correlation between the two traits. We have estimated the heritability of both traits and the genetic correlation between them. Estimating the quantitative genetic parameters associated with ecologically important traits is crucial to the understanding and modeling of life-history evolution. Note that this paper does not deal with the frequent association between reproductive diapause and migration, or oogenesis-flight syndrome, found in many insect species (eg, Dingle, 1996).

The spring field cricket G. veletis (Alexander and Bigelow, 1960) can be found in north-eastern USA and along the southern part of Canada, and lives in a variety of grassy areas. Gryllus veletis enters a cold-resistant nymphal diapause in the fall and remains in diapause until the following spring. Diapause allows this species to synchronize its reproductive stage with late spring/early summer, producing a single generation a year. Gryllus veletis is wing dimorphic but the proportion of macropterous individuals in nature is usually under five percent (Alexander, 1968). Laboratory experiments performed by Alexander (1968) showed that wing morphology and diapause occurrence phenotypically covary in this species, making it ideal for the investigation of the genetic basis of these two traits.

\section{Materials and methods}

\section{Experimental protocol}

Approximately 200 late instar nymphs of G. veletis were collected in a field near Montréal, Québec, Canada in early May 1999. These immature individuals were brought into the lab and separated according to sex to avoid uncontrolled matings. After emergence of the adults, pairs were randomly formed and put into plastic containers with an unlimited amount of water and food (rabbit chow) and a dish of moist earth for oviposition. The plastic containers were stored in a growth chamber at a constant temperature $\left(28^{\circ} \mathrm{C}\right)$ and relative humidity (50\%), and a photoperiod of $15 \mathrm{~h}$ of light for $9 \mathrm{~h}$ of darkness. Twelve days after a parental pair was formed, the earth dish was removed and put into a separate plastic container to facilitate the collection of nymphs. A total of 80 newly hatched offspring were collected from each family. If the number of offspring was not sufficient, the nymphs were disposed of and the whole nymph collection process was repeated. Newly hatched nymphs were collected within three days to avoid large differences in size between siblings. Nymphs were stored in two 4 litre plastic buckets, at a density of 40 per bucket, with environmental conditions identical to those experienced by the parental generation in the laboratory. These rearing conditions were maintained for a maximum of 79 days, which was sufficient for all direct developing crickets to emerge as adults. However, a substantial number of individuals did not fully develop and instead entered a nymphal diapause. To break their diapause and allow these nymphs to continue their development, the temperature was decreased to $4^{\circ} \mathrm{C}$ to simulate winter, using decrements of $4^{\circ} \mathrm{C}$ per 3 days. The temperature was maintained at $4^{\circ} \mathrm{C}$ for 107 days and then brought back up to $28^{\circ} \mathrm{C}$ using increments of $4^{\circ} \mathrm{C}$ per 3 days. This treatment allowed most of the diapausing crickets to complete their development. Throughout this experiment, individuals were killed and preserved within 2 days after reaching adulthood.

A total of 81 full-sib families were produced, with two cages per family. The proportion of survivors to adulthood was $53 \%$, for a total of 3446 scored adults. The winter simulating period imposed on the crickets might explain this relatively low survival proportion. This hypothesis is supported by a significant negative correlation between the proportion of diapausing individuals in a cage and the survival proportion within that same cage $(r=-0.50$; d.f. $=79 ; P<0.001)$. For logistical reasons, it was impossible to keep track of the mortality of specific individuals. This problem has two main consequences. First, survival could not be analysed in relation to diapause occurrence and wing morphology. Second, the trait 'diapause occurrence' is really 'diapause occurrence of survivors' because individuals that died as nymphs during the diapause process could not be scored as diapausers. The proportion of diapausing individuals reported in this study is therefore underestimated. Because of the genetic correlation between diapause occurrence and wing morphology found in this study (see Results section), the same is true for the trait 'wing morphology'; the proportion of micropterous individuals is probably underestimated.

A second sampling of individuals from the wild was made in June 1999 in the same location (see above). The purpose was to collect adults from the field and compare them to laboratory reared individuals. A total of 107 adult $G$. veletis were collected and scored for wing morphology and diapause occurrence (all were obviously diapausers because direct developers would inevitably 
die during the winter). Note that collecting adults in the field might produce an underestimation of the proportion of macropterous individuals because these can potentially fly away, leaving a greater proportion of micropterous adults and immature individuals. However, this bias is likely to be small because our estimation of the proportion of the two wing morphs is concordant with the literature (see the Results and Discussion sections) and because macropterous insects coming from a population with a low proportion of macroptery are known to have a low propensity to fly (Fairbairn and Roff, 1990).

In addition to wing morphology and diapause occurrence, five morphological traits were measured on both laboratory and field individuals to provide an estimate of adult size in both environments. The five traits were head width, prothorax width, prothorax length, femur length and ovipositor length. To avoid any possible comparison bias arising from size differences between sexes or between morphs, only females that were both micropterous and diapausing were measured (54 laboratory reared females and 57 field caught females). This particular morph was chosen to maximize sample size, as it represents the most frequent combination in the field sample. The laboratory reared and field caught crickets were compared using a MANOVA. The univariate normality of the distribution of all five traits was tested for both laboratory and field samples using the one-sampleKolmogorov-Smirnov test (Lilliefors option). The results (not shown) indicate that nine trait distributions out of ten did not depart significantly from normality, the sole exception being ovipositor length in the field population. There is no guarantee that univariate normality of all variables produces multivariate normality. However, the normality of each variable and the generally oval shape of most bivariate trait distributions suggest that deviation from multivariate normality should not be very large, if it exist. With more than 100 degrees of freedom for error for each variable in this analysis (see Results section), a multivariate analysis of variances is known to be generally robust to deviations from multivariate normality provided that there are no outliers. We tested for multivariate outliers using the Mahalanobis distance and found six marginally significant outliers. We ran the MANOVA without these outliers and found that the statistics of the test were very similar to the results that include the outliers. For these reasons, we chose to keep all the data and chose not to transform it.

\section{Statistical analysis}

The analysis of threshold traits assumes that some unmeasured trait, called the liability, is normally distributed in the population and controls for the dichotomous variation of the observed trait. A fixed threshold value for the liability is assumed to exist in the population, with each individual being of one morph or the other depending on its liability value (Falconer and Mackay, 1996). These assumptions allow the use of standard quantitative genetic methods for the analysis of dichotomously varying traits that have a polygenic basis. During this study, every individual that completed its development was scored for wing morphology $(0=$ macropterous; 1 = micropterous) and occurrence of diapause ( $0=$ direct development; $1=$ diapause). Heritabilities on the $0 / 1$ scale were derived from the results of a nested ANOVA, with 'family' and 'cage nested within

family' as the two independent variables (Roff, 1997).

Standard errors were estimated using the approximate formula for unequal family sizes developed by Swiger, Harvey, Everson and Gregory (p 42 of Roff, 1997). To obtain the corresponding estimates on the liability scale of the threshold traits, both heritabilities and their standard errors where transformed using the formula developed by Dempster and Lerner (p 55 of Roff, 1997) which corrects for the observed frequency of each morph. All heritabilities and their standard error given in this paper correspond to the liability, not the $0 / 1$ scale. The estimates for the genetic correlation and its standard error were obtained using a nested ANCOVA followed by a Jackknife procedure (Roff, 1997).

Estimating additive genetic parameters using a nested full-sib family breeding design introduces two potential sources of bias: dominance effects and maternal effects. For this reason, the estimates provided in this paper have to be interpreted as upper limit estimates. However, Roff (1986b, 1990) showed that maternal effects and non-additive genetic effects, respectively, are not important sources of bias in the estimation of the heritability of wing morphology in G. firmus, a closely related species. Unfortunately, nothing is known about the effect of these sources of variation in the estimation of the heritability of nymphal diapause.

\section{Results}

\section{Comparison of laboratory reared and field caught crickets}

The adult size of field caught and laboratory reared crickets were first compared, using only females that underwent diapause and were micropterous (see Materials and methods section). A MANOVA was performed using the five morphological traits as dependent variables, and rearing conditions (laboratory or field) as the independent variable. The results revealed a significant multivariate difference between laboratory and field individuals (Wilk's $\lambda=0.351$; approx. $F=38.8$; d.f. $=5$, 105; $P<0.001)$ and a significant univariate difference for all five traits (results of the individual $F$ tests not shown but $P$ varied between 0.05 and $<0.001$ ). This constant and unidirectional difference can be observed in the trait means, which were between $3 \%$ and $11 \%$ larger in the laboratory population compared to the field population (Table 1). Second, field caught and laboratory reared

Table 1 A comparison of laboratory reared and field caught adult crickets. Measurements are in $\mathrm{mm}$ and standard deviations are in brackets. All five traits are significantly larger in the laboratory sample. These morphological measurements were taken on micropterous diapausing females only. Percentages were calculated based on all available individuals

\begin{tabular}{lcc}
\hline Measurement & Field & Laboratory \\
\hline Head width & $4.76(0.24)$ & $5.07(0.22)$ \\
Prothorax length & $3.23(0.19)$ & $3.55(0.19)$ \\
Prothorax width & $5.11(0.25)$ & $5.67(0.25)$ \\
Femur length & $9.42(0.44)$ & $10.11(0.48)$ \\
Ovipositor length & $12.27(0.78)$ & $12.71(1.45)$ \\
Percentage of micropters & $95.3 \%$ & $6.4 \%$ \\
Percentage of diapausers & $100 \%$ & $26.2 \%$
\end{tabular}


crickets, using all crickets available, were compared for proportion of diapause occurrence and wing dimorphism. Field caught individuals were mostly micropterous (102 out of 107) and all had successfully completed diapause (Table 1). Laboratory reared crickets were mostly macropterous (3224 out of 3446) and non diapausing (2542 out of 3446) (Table 1 ).

\section{Heritabilities}

The estimate of the heritability of wing morphology was 0.25 with a standard error of 0.09 . This heritability value was significantly different from 0 , as shown by the $\mathrm{F}$ value corresponding to the family effect in the ANOVA ( $F=1.96$; d.f. $=80,81 ; P=0.001)$. The cage effect for this trait was also significant $(F=1.57$; d.f. $=81,3282 ; P=$ 0.001). The estimate of the heritability of diapause occurrence was 0.77 with a standard error of 0.11 . Both the family effects $(F=7.49$; d.f. $=80,81 ; P<0.001)$ and the cage effects $(F=1.81$; d.f. $=81,3282 ; P<0.001)$ were significant.

\section{Genetic correlation}

The majority (90\%) of the micropterous individuals underwent diapause, whereas only $22 \%$ of the macropterous individuals did so. These results suggest a correlation between wing morphology and diapause occurrence, which was confirmed by an estimation of the phenotypic correlation of 0.38 with a standard error of 0.02 and by an estimation of the genetic correlation of 0.61 with a standard error of 0.19 . This genetic correlation was both different from $0(t=3.25$; d.f. $=80 ; P=0.017)$ and from 1 ( $t=2.08$; d.f. $=80 ; P=0.04)$. With respect to our coding, a positive correlation coefficient indicates that families with a high genetic predisposition towards a direct development also tend to have a high genetic predisposition towards macroptery. The point estimate of the genetic correlation obtained after applying the Jack-knife (0.61) was in agreement with the original estimate from the ANCOVA (0.62), providing support for the use of the Jack-knife in the estimation of genetic correlations (Roff and Preziosi, 1994).

\section{Effect of gender}

The percentage of micropterous individuals and of diapausing individuals in the whole laboratory sample differed across sexes, males being more often micropterous and diapausing than females (Table 2). The statistical

Table 2 Summary of the analysis of wing morphology and diapause occurrence for the pooled sample and for the males and females separately. Quantitative genetic parameters are given with their standard error. The heritabilities were estimated based on a nested ANOVA and the genetic correlation was estimated by applying a Jackknife procedure on the ANCOVA results

\begin{tabular}{lccc}
\hline Estimates & $\begin{array}{c}\text { Pooled } \\
\text { sample }\end{array}$ & Males only & Females only \\
& & \\
\hline $\begin{array}{l}\text { Percentage of micropters } \\
\text { Percentage of diapausers }\end{array}$ & $6.4 \%$ & $8.2 \%$ & $4.6 \%$ \\
$\begin{array}{l}\text { Heritability of wing } \\
\text { morphology }\end{array}$ & $0.25(0.09)$ & $0.28(0.13)$ & $\begin{array}{r}\text { NA* } \\
\text { Heritability of diapause }\end{array}$ \\
$\begin{array}{l}\text { Phenotypic correlation } \\
\text { Genetic correlation }\end{array}$ & $0.77(0.11)$ & $0.78(0.13)$ & $1.00(0.16)$ \\
& $0.61(0.19)$ & $0.42(0.03)$ & $0.29(0.03)$ \\
\hline
\end{tabular}

*The estimate of family effect in the ANOVA was negative (see Discussion section). significance of this difference was tested using a $2 \times 2$ contingency table analysis with sex and wing morphology or diapause occurrence as variables. This analysis revealed that proportions of wing dimorphism $\left(\chi^{2}=\right.$ 18.87; d.f. $=1 ; P<0.001)$ and of diapause occurrence $\left(\chi^{2}\right.$ $=98.31$; d.f. $=1 ; P<0.001)$ differed across sexes. However, this analysis does not take into account the family and cage structure of the data. It was not possible to do so with this type of analysis because many cages have a count of 0 in the cells corresponding to 'micropters' and 'diapausers' thus producing too many undefined $\chi^{2}$ values. To incorporate the cage structure, a paired-sample $t$-test was done using the proportion per cage of a particular morph. The proportion of wing dimorphism was found to differ across sexes $(t=3.792 ;$ d.f. $=161 ; P$ $<0.001)$ and so did the proportion per cage of diapause occurrence $(t=8.980$; d.f. $=161 ; P<0.001)$. The results of this analysis thus support the results of the contingency table analysis. Because sex had an effect on the proportions of diapause occurrence and wing dimorphism, quantitative genetic parameters were estimated separately for each sex.

In males, the estimates of heritability and of the genetic correlation were found to be extremely similar to the pooled estimates (Table 2). The heritability of wing morphology was significantly different from 0 ( $F=1.65$; d.f. $=80,81 ; P=0.013)$, the heritability of diapause occurrence was different from $0(F=5.35$; d.f. $=80,81 ; P<0.001)$ and the genetic correlation was different from both $0(t$ = 3.77; d.f. $=80 ; P<0.001)$ and $1(t=2.12$; d.f. $=80 ; P=$ 0.037). To test for a significant difference between the male estimate and the pooled estimate, a Jack-knife procedure was used to provide a distribution of heritability (or genetic correlation) and a $t$-test was then implemented to compare the two groups. The heritability of wing morphology in males was not significantly different from the pooled sample estimate $(t=0.15$; d.f. $=160$; $P=0.880$ ), the heritability of diapause occurrence was not different from the pooled estimate $(t=0.11$; d.f. $=160 ; P$ $=0.909$ ) and the genetic correlation did not differ from the estimate of the pooled data set $(t=0.15$; d.f. $=160 ; P$ $=0.881$ ).

In females, the heritability of diapause occurrence was different from 0 ( $F=5.32$; d.f. $=80,81 ; P<0.001)$ and was not significantly different from the pooled estimate $(t=1.26$; d.f. $=160 ; P=0.210)$ (Table 2$)$. However, the heritability of wing morphology could not be estimated because the ANOVA gave a non-significant negative estimate of the family effect $(F=0.88$; d.f. $=80,81 ; P=0.72)$. Because the calculations of the heritability of a threshold trait require the initial heritability on the $0 / 1$ scale to be multiplied by a function of the proportions of the morphs, it made no sense to complete this procedure and obtain a higher negative estimate (but see the Discussion section). Similarly the genetic correlation using only females could not be estimated.

\section{Discussion}

\section{Effect of gender}

The analysis of the effect of gender on wing morphology and diapause occurrence revealed that females have a higher tendency to be macropters and to be direct developers than males (Table 2). This tendency has also been 
observed for wing morphology in three congeners; $G$. rubens (Veazey et al., 1976; Walker, 1987), G. firmus (Roff, 1986b) and G. pennsylvanicus (Harrison, 1979) but does not hold for insects in general (Roff, 1986b). In the case of wing dimorphism, the difference between sexes in the proportions of the two wing morphs is probably maintained by the differences between the two sexes in the fitness costs associated with possessing a functional flight apparatus (reviewed in Roff and Fairbairn, 2001). However, the difference between sexes in the proportion of diapausers is probably an artifact of the laboratory because all $G$. veletis individuals undergo diapause in nature.

The differences between sexes in proportions of wing dimorphism and diapause occurrence in G. veletis suggest that heritabilities and genetic correlations might also differ between males and females, which would suggest that these parameters should be estimated for each sex separately. However, the results of this analysis suggest that this is not necessary. In the case of males, the point estimates for the three parameters are remarkably similar to the ones for the pooled sample (Table 2). For females, the differences are more marked than in males but are still relatively small. However, the case of the heritability of wing morphology deserves some attention. The non significant negative family effect revealed by the ANOVA is in fact very small and is probably simply caused by statistical noise. It is relevant to consider the heritability of wing morphology on the original $0 / 1$ scale (before conversion to the liability scale): the estimate for the pooled sample on the $0 / 1$ scale was $0.07 \pm 0.02$ whereas the female estimate was $-0.02 \pm 0.03$. With a different sample of crickets, the female heritability could easily have been slightly positive, in which case the pooled and female estimates would not have been significantly different. Overall the results suggest that there is not a major difference between sexes with respect to heritabilities and genetic correlation, and that estimating these genetic parameters with a pooled sample is reasonable (note however that the standard errors around these quantitative genetic parameters are relatively large, making the power of these tests rather low). Similarly Roff (1986b) showed that the heritability of wing dimorphism does not differ across sexes in the cricket G. firmus.

\section{Comparison of laboratory reared and field caught crickets}

The comparison of laboratory reared with field caught individuals revealed that the environment has a substantial effect on three aspects of the development of $G$. veletis. First, the laboratory environmental regime seemed to be favourable to growth. The average measure of each of the five morphological traits of laboratory reared adults was significantly larger than that of field caught adults (Table 1). This result is similar to the one presented in Simons and Roff (1994) for the closely related cricket G. pennsylvanicus but is opposite to the findings of Mousseau and Roff (1989) for the cricket Allonemobius fasciatus. Second, the percentage of macropterous individuals was dramatically different in the two environments, laboratory reared crickets being mostly macropterous whereas field crickets were mostly micropterous (Table 1). Our estimation of the proportion of macroptery in nature $(4.7 \%)$ is in agreement with the literature (4\%, Alexander, 1968), indicating that the high proportion of macropter-

ous individuals in the laboratory can be attributed to the

laboratory environment and not to the studied population. Third, the proportion of diapausing individuals in the laboratory was relatively low $(26.2 \%)$ whereas field caught individuals all underwent diapause. Our estimation of the proportion of diapausing individuals in the laboratory is in agreement with the experiments of Alexander and Bigelow (1960) who found that, in similar laboratory conditions, approximately $25 \%$ of the individuals entered diapause. The relatively low proportion of diapausers in the laboratory suggests that these conditions did not correspond to the environmental cues that $G$. veletis individuals need to enter diapause, these conditions being, among others, a decreasing and $\backslash$ or shorter photoperiod (Tanaka, 1978; Tauber et al, 1986; Masaki and Walker, 1987). All three aforementioned lines of evidence clearly indicate that growth, wing morphology and diapause occurrence are all highly plastic traits in $G$. veletis. This result is compatible with other laboratory studies that have demonstrated the plasticity of diapause occurrence and of wing dimorphism in other cricket species (eg, Tanaka, 1978).

\section{Heritabilities}

It was not surprising to find that both wing morphology and diapause occurrence are heritable in $G$. veletis because these two traits have been found to be heritable in many species of arthropods (reviewed in Roff, 1996; Roff and Fairbairn, 2001). In particular a significant heritability of wing morphology has been estimated in three congeners; G. firmus (Roff, 1986b), G. rubens (Roff and Fairbairn, 1991), and G. pennsylvanicus (Roff and Simons, 1997). The presence of genetic variation in wing morphology and the adaptive value of this trait in crickets have been abundantly studied (eg, Roff and Fairbairn, 1991; Roff, 1994a, b) and will not be discussed here. On the other hand the heritability of diapause occurrence was estimated here for the first time in a species of the genus Gryllus. Contrary to the case of wing morphology in which maternal effects and non-additive genetic effects are not important sources of variation (Roff, 1986b, 1990), it is not known if this estimate of the heritability of diapause occurrence is inflated by these two effects. Maternal effects are known to be important in the case of egg diapause in bivoltine populations of the cricket $A$. fasciatus (Mousseau, 1991). However, nymphal diapause is inherently less influenced by the mother than is egg diapause and $G$. veletis is an obligate diapauser in the wild, making the presence of strong maternal effects less likely.

The heritability value for nymphal diapause occurrence in $G$. veletis is particularly interesting because it indicates that genetic variation in this trait is abundant in this species despite natural populations not being phenotypically variable. A significant laboratory heritability of egg diapause occurrence has also been observed in populations of the cricket $A$. socius that are monomorphic for diapause in the wild (Mousseau and Roff, 1989). This situation can be explained by invoking a highly plastic response of the liability of this threshold trait. In nature the environment causes the mean liability value of the population to be far from the threshold value, hence preventing the production of the direct developing morph and hiding any genetic variation. Conversely the laboratory conditions cause the liability distribution to 
overlap the threshold, a situation that produces both morphs and allows the quantification of genetic variation. For this reason the estimate of the heritability of diapause occurrence found in the laboratory is not informative with respect to the potential for evolution of this trait in natural populations of G. veletis. However, if populations of this species were to colonize habitats with different environmental conditions, genetic variation for diapause occurrence might become expressed, which could potentially allow this trait to evolve. On the other hand we believe that the evolution of the expression of diapause is more likely to occur through the evolution of the reaction norm of this trait. This situation is likely to have occurred in the cricket $A$. socius in which genetic differences in the reaction norm of diapause occurrence have been shown between populations along a latitudinal gradient (Bradford and Roff, 1995).

\section{Genetic correlation}

The genetic correlation between wing morphology and diapause occurrence estimated in this study was moderately high (0.61) and indicates that, in the cricket $G$. veletis, these two traits share part of their genetic basis. The present paper is the first in which this genetic correlation is estimated. This finding supports the previous observation of a phenotypic association between these two traits in G. veletis (Alexander, 1968). The common genetic basis of wing morphology and diapause occurrence in crickets is probably expressed as a complex hormonal pathway which partly determines the phenotype of both of these traits (Sellier, 1954; Tanaka, 1978). Indeed some insect hormones, such as the juvenile hormone, have been shown to have an overall effect on most aspects of insect development (Nijhout, 1994). However, at present, it is not possible to link a specific hormone with the common developmental basis of wing morphology and diapause occurrence.

The evolutionary significance of the genetic correlation between these two traits is not obvious in G. veletis because natural populations of this species are monomorphic for diapause occurrence, and hence this correlation is not expressed in nature. However, the quantitative genetic information obtained in the present study can be used to shed light on the life cycle of other nymph diapausing and wing dimorphic cricket species. Alexander (1968) and Veazy et al, (1976) report the example of $G$. rubens, a bivoltine cricket. In this species the spring peak of adults, originating from diapausing nymphs, is mostly micropterous whereas the summer peak, produced without diapause, consists of significantly more macropters. This observation suggests a trade-off between the ability to disperse by flight and the ability to diapause. If the genetic correlation found in G. veletis is also present in this closely related species, which is likely considering the similarities, the trade-off is genetically based. This postulated genetic trade-off may have acted as a genetic constraint to the independent evolution of diapause and dispersal by flight in this species, contributing to its adaptation to both spatial and temporal environmental heterogeneity. Such a trade-off might be present in all cricket species in which wing morphology and nymphal diapause occurrence have been shown to phenotypically covary: G. campestris (Sellier, 1954), G. rubens (Alexander, 1968), G. texensis (incorrectly referred to as G. integer in Alexander, 1968) G. veletis (this study) and
Pteronemobius nitidus (Tanaka, 1978). Additionally it would be interesting to know if a common genetic basis also controls for the expression of both diapause occurrence and wing dimorphism in Hemipterans, the other insect order in which a phenotypic association between these two traits has been observed. In this group of insects, however, the genetic and physiological basis of these traits could be very different because diapause occurs in the adult stage and it is the macropterous morph that is associated with the diapausing stage (Harrison, 1980; Harada and Numata, 1993).

A few studies have estimated genetic correlations between one of these two traits and various other traits in insects. Diapause occurrence has been found to be genetically correlated with developmental time in the mosquito Wyeomyia smithii (Scheiner and Istock, 1991) and in the flesh fly Sarcophaga bullata (Henrich and Denlinger, 1982), with body size in the cricket $A$. fasciatus (family mean correlation; Mousseau and Roff, 1989), and with larval weight in the moth Choristoneura rosaceana (Carriè re and Roff, 1995). Wing morphology has been found to be genetically correlated with body size in the bug Oncopeltus fasciatus (Palmer and Dingle, 1986) and in the cricket A. socius (Roff and Bradford, 1998), with developmental time in the bug O. fasciatus (Palmer and Dingle, 1986) and in the cricket G. firmus (Roff, 1995) and with early fecundity in O. fasciatus (Palmer and Dingle, 1986), in G. firmus (Roff, 1995) and in A. socius (Roff and Bradford, 1996). These studies provide evidence that, across four different insect orders, diapause occurrence and wing morphology are genetically associated with several ecologically important traits and should therefore be studied as part of the whole life history of these insects.

\section{Acknowledgements}

We wish to thank Denis Réale and two anonymous reviewers for helpful comments, Émile Geoffroy for laboratory assistance and McGill University for the opportunity to sample at the Gault estate. This work was supported by post-graduate scholarships from NSERC (Canada) and FCAR (Québec) to Mattieu Bégin and by a NSERC operating grant to Derek A Roff.

\section{References}

Alexander RD (1968). Life cycle origins, speciation, and related phenomena in crickets. $Q$ Rev Biol 43: 1-41.

Alexander RD, Bigelow RS (1960). Allochronic speciation in field crickets, and a new species, Acheta veletis. Evolution 14: $334-$ 346.

Bradford MJ, Roff DA (1995). Genetic and phenotypic sources of life history variation along a cline in voltinism in the cricket Allonemobius socius. Oecologia 103: 319-326.

Carrière Y, Roff DA (1995). Change in genetic architecture resulting from the evolution of insecticide resistance: a theoretical and empirical analysis. Heredity 75: 618-629.

Danks HV (1987). Insect Dormancy: an Ecological Perspective. Biological Survey of Canada: Ottawa.

Dingle H (1978). Evolution of Insect Migration and Diapause. Springer-Verlag: New York.

Dingle H (1996). Migration. Oxford University Press: New York.

Fairbairn DJ, Roff DA (1990). Genetic correlations among traits determining migratory tendency in the sand cricket, Gryllus firmus. Evolution 44: 1787-1795.

Falconer DS, Mackay TFC (1996). Introduction to Quantitative Genetics. Longman: Harlow, England. 
Harada T, Numata H (1993). Two critical day lengths for the determination of wing forms and the induction of adult diapause in the water strider, Aquarius paludun. Naturwissenschaften 80: 430-432.

Harrison RG (1979). Flight polymorphism in the field cricket Gryllus pennsylvanicus. Oecologia 40: 125-132.

Harrison RG (1980). Dispersal polymorphisms in insects. Annu Rev Ecol Syst 11: 95-118.

Henrich VC, Denlinger DL (1982). Selection for late pupariation affects diapause incidence and duration in the flesh fly, Sarcophaga bullata. Physiol Entomol 7: 407-411.

Masaki S, Walker TJ (1987). Cricket life cycles. Evol Biol 21 349-423.

Mousseau TA (1991). Geographic variation in maternal-age effects on diapause in a cricket. Evolution 45: 1053-1059.

Mousseau TA, Roff DA (1989). Adaptation to seasonality in a cricket: patterns of phenotypic and genotypic variation in body size and diapause expression along a cline in season length. Evolution 43: 1483-1496.

Nijhout HF (1994). Insect Hormones. Princeton University Press: Princeton, NY.

Palmer JO, Dingle H (1986). Direct and correlated responses to selection among life-history traits in milkweed bugs (Oncopeltus fasciatus). Evolution 40: 767-777.

Roff DA (1986a). The evolution of wing dimorphism in insects. Evolution 40: 1009-1020.

Roff DA (1986b). The genetic basis of wing dimorphism in the sand cricket, Gryllus firmus and its relevance to the evolution of wing dimorphisms in insects. Heredity 57: 221-231.

Roff DA (1990). Selection for changes in the incidence of wing dimorphism in Gryllus firmus. Heredity 65: 163-168.

Roff DA (1994a). Habitat persistence and the evolution of wing dimorphism in insects. Am Nat 144: 772-798.

Roff DA (1994b). Why is there so much genetic variation for wing dimorphism? Res Popul Ecol 36: 145-150.

Roff DA (1995). Antagonistic and reinforcing pleiotrophy: a study of differences in development time in wing dimorphic insects. J Evol Biol 8: 405-419.

Roff DA (1996). The evolution of threshold traits in animals. $Q$ Rev Biol 71: 3-35.

Roff DA (1997). Evolutionary Quantitative Genetics. Chapman and Hall: New York.

Roff DA (2002). Life History Evolution. Sinauer Associates: Sunderland, MA, USA

Roff DA, Bradford MJ (1996). Quantitative genetics of the tradeoff between fecundity and wing dimorphism in the cricket Allonemobius socius. Heredity 76: 178-185.

Roff DA, Bradford MJ (1998). The evolution of shape in the wing dimorphic cricket, Allonemobius socius. Heredity 80: 446-455.

Roff DA, Fairbairn DJ (1991). Wing dimorphisms and the evolution of migratory polymorphisms among the Insecta. Am Zool 31: 243-251.

Roff DA, Fairbairn DJ (2001). The genetic basis of dispersal and migration, and its consequences for the evolution of correlated traits. In: Clobert J, Danchin E, Dhondt AA, Nichols JD (eds). Dispersal, Oxford University Press: Oxford, pp 191-202.

Roff DA, Preziosi R (1994). The estimation of the genetic correlation: the use of the jackknife. Heredity 73: 544-548.

Roff DA, Simons AM (1997). The quantitative genetics of wing dimorphism under laboratory and 'field' conditions in the cricket Gryllus pennsylvanicus. Heredity 78: 235-240.

Scheiner SM, Istock CA (1991). Correlational selection on life history traits in the pitcher-plant mosquito. Genetica 84: 123128.

Sellier R (1954). Recherches sur la morphogénèse et le polymorphisme alaires chez les orthoptères gryllides. Ann Sci Nat Zool 11 ser 16: 595-740.

Simons AM, Roff DA (1994). The effect of environmental variability on the heritabilities of traits of a field cricket. Evolution 48: 1637-1649.

Tanaka S (1978). Photoperiodic determination of wing form in Pteronemobius nitidus Bolivar (Orthoptera, Gryllidae). Kontyu, Tokyo 46: 207-217.

Tauber MJ, Tauber CA, Masaki S (1986). Seasonal Adaptations of Insects. Oxford University Press: New York.

Veazey JN, Kay CAR, Walker TJ, Whitcomb WH (1976). Seasonal abundance, sex ratio, and macroptery of field crickets in Northern Florida. Ann Entomol Soc Am 69: 374-380.

Walker TJ (1987). Wing dimorphism in Gryllus rubens (Orthoptera: Gryllidae). Ann Entomol Soc Am 80: 547-560.

Zera AJ, Denno RF (1997). Physiology and ecology of dispersal polymorphism in insects. Annu Rev Ent 42: 207-230. 